Nouvelles perspectives en sciences sociales

\title{
Agent_Zero: Toward Neurocognitive Foundations for Generative Social Science, Joshua Epstein, Princeton (NJ), Princeton University Press, 2013
}

\section{Oswaldo Teran}

Volume 12, numéro 1, novembre 2016

URI : https://id.erudit.org/iderudit/1038378ar

DOI : https://doi.org/10.7202/1038378ar

Aller au sommaire du numéro

Éditeur(s)

Prise de parole

ISSN

1712-8307 (imprimé)

1918-7475 (numérique)

Découvrir la revue

Citer ce compte rendu

Teran, O. (2016). Compte rendu de [Agent_Zero: Toward Neurocognitive Foundations for Generative Social Science, Joshua Epstein, Princeton (NJ),

Princeton University Press, 2013]. Nouvelles perspectives en sciences sociales, 12(1), 265-268. https://doi.org/10.7202/1038378ar d'utilisation que vous pouvez consulter en ligne. 


\title{
Agent_Zero: Toward Neurocognitive Foundations for Generative Social Science
}

Joshua Epstein, Princeton (NJ), Princeton University Press, 2013.

\author{
PAR OSWALDO TERAN \\ Université de Los Andes, Venezuela \\ Traduit de l'espagnol par Leonardo G. Rodriguez Zoya
}

\section{D} ans ce travail, Joshua Epstein continue le projet d'une science sociale générative, initié il y a vingt ans avec la parution de Growing Artificial Societies; et poursuivi avec Generative Social Science; où l'auteur développe avec plus de profondeur la modélisation à base d'agents en économie, archéologie, épidémiologie, entre autres champs des sciences sociales. Agent_ Zéro constitue la troisième partie de cette trilogie pionnière dans le domaine de la simulation sociale, dont la particularité distinctive est d'analyser et d'intégrer les fondements neurocognitifs d'une science sociale générative.

Avec Agent_Zéro, il s'agit d'une nouvelle entité théorique concrétisée dans un modèle de simulation sociale qui tient compte des aspects émotionnels, cognitifs et sociaux du comportement et de la prise de décision. Ce modèle a la prétention d'être minimaliste et de portée générale, c'est-à-dire d'être un modèle simple qui est utile pour analyser des phénomènes sociaux divers, tels qu'un génocide ou une panique financière. En outre, il se veut génératif, souhaitant simuler l'émergence de dynamiques sociales de bas en haut.

L'ouvrage est organisé en quatre parties. La première introduit le modèle mathématique et décrit les composants émotionnels, rationnels et sociaux intervenant dans le processus de la prise de décision. La deuxième développe la modélisation à base d'agents sur la plateforme NetLogo et présente la simulation de quelques scénarios relativement simples. Sur cette base, la troisième partie 
aborde plusieurs prolongements possibles du modèle. Enfin, la quatrième partie, suggère des lignes de travail pour l'avenir et donne dans des annexes le code mathématique et numérique du modèle, ce qui semble utile pour des finalités pratiques et pédagogiques.

Nous nous arrêtons sur certains aspects du travail d'Epstein.

D'abord, dans la première partie, l'auteur essaie de construire un modèle intégrant les aspects cognitifs, émotionnels et sociaux de l'action. Pour atteindre cet objectif, il propose, d'un côté, une systématisation théorique des contributions des neurosciences à la compréhension de la prise de décision et de l'action humaine. D'un autre côté, il reprend un modèle classique du conditionnement développé au début des années 1970 par Robert A. Rescorla et Allan R. Wagner;. Il s'agit d'un modèle formel, à base mathématique, qui repose sur des expériences en laboratoire et dont le but est de simuler un processus d'apprentissage de type pavlovien.

Le travail d'Epstein soulève deux questions. La première se révèle dans la considération de la peur comme expression prototypique du composant émotionnel du modèle. L'auteur soutient que les conclusions du modèle Rescorla-Wagner, développé dans contexte précis, sont valides pour d'autres domaines. La seconde faiblesse apparait évidente dans l'absence d'explication satisfaisante sur le lien entre le modèle Rescorla-Wagner et les contribuions des neurosciences. À la limite, on constante une brèche argumentative entre la discussion théorique sur la cognition et le cerveau, d'un part, et la construction d'un modèle fondé sur une équation issue de la psychologie expérimentale, de l'autre. Autrement dit, contrairement à ce qu'affirme l'auteur, le modèle n'est pas fondé sur les neurosciences cognitives mais sur un modèle classique de conditionnement du comportement.

En ce qui concerne la simulation numérique, implantée sur NetLogo et développée dans la deuxième partie, il faut souligner la manière dont l'auteur traite les trois composants d'un modèle à base d'agents : les agents, l'environnement et les règles d'interaction. D'abord, il est vraiment étonnant que le modèle ne prennent en considération que trois agents, ce qui semble un 
nombre très limité étant donné la prétention de simuler l'émergence de dynamiques sociales complexes. Par rapport à la représentation de l'environnement, Agent_Zéro, est se fonde sur un espace cellulaire de type treillis, même si des dispositions plus sophistiquées, comme celle de taureau, sont disponibles sur NetLogo. Chaque cellule peut avoir deux états : active (en orange) ou inactive (en jaune). Quand une cellule est inactive, elle est considérée neutre, tandis que si elle est active, elle entraîne des conséquences, soit positives, soit négatives, pour les agents qui les occupent. Les cellules peuvent être mobilisées pour représenter différents phénomènes, tels que l'activité d'une population sur un territoire, les conséquences des vaccins, des actions boursières, l'expérience musicale, entre autres. Finalement, l'architecture cognitive des agents se base sur un algorithme décisionnel intégrant les aspects émotionnels, rationnels et sociaux, tous étant représentés par un nombre réel. Les trois valeurs sont additionnées et le chiffre qui en résulte est comparé avec un seuil pour décider si l'agent agit ou non. Dans le cas où le chiffre est supérieur au seuil, l'agent prend la décision d'agir, tandis que, dans le cas contraire, il opte pour ne pas agir.

Dans la troisième partie, Epstein explore quatorze prolongements possibles d'Agent_Zéro, parmi lesquels il faut souligner l'enchevêtrement entre émotion et raison. Au lieu de considérer ces deux composants de manière indépendante, la valeur de la rationalité est modifiée par une fonction exponentielle de l'émotion, de telle manière que si la valeur de ce dernier aspect est nulle, la rationalité demeure inaltérée. La simulation du printemps arabe de 2011 constitue un autre prolongement intéressant. Dans ce cas, les cellules actives (en orange) représentent des faits de corruption du gouvernement diffusés par les médias, face auxquels les agents réagissent. L'émotion des agents augmente alors qu'ils se déplacent, ce qui génère un réseau social émergeant dont le comportement modifie l'environnement. De cette manière, la simulation permet d'explorer la chute d'un gouvernement et l'émergence du printemps arabe. 
Pour conclure cette discussion critique du travail d'Epstein, il faudrait souligner qu'il s'agit d'un ouvrage captivant dont la contribution majeure est de jeter un pont entre les sciences sociale et les neurosciences cognitives dans une perspective qui mobilise la simulation numérique comme un outil méthodologique pertinent pour la recherche sociologique. Néanmoins, un des points faibles d'Agent_Zéro, c'est son manque de données empiriques pour valider les modèles. Par conséquence, les scénarios simulés ont peu de relation avec des cas réels. En outre, l'algorithme décisionnel du modèle simplifie un peu trop les situations de sorte qu'il ne semble pas vraiment pertinent pour représenter la complexité du comportement humain. Dans ce sens, il faudrait souligner les contributions pionnières d'Herbert A. Simon; sur la rationalité limitée, contributions qui ne sont pas vraiment prises en compte par Agent_Zéro. Pour être précis, dans le modèle d'Epstein, la rationalité limitée est modélisée comme un biais dans la prise de décision tandis que le composant social influant sur l'agent n'a pas une portée locale mais globale, c'està-dire qu'il comprend tout l'espace simulé. Il faudrait donc doter Agent_Zéro d'un mécanisme décisionnel plus robuste en termes tant de validité empirique que de fondements théoriques.

En bref, le livre déçoit dans un bilan global, car il ne réussit pas à atteindre l'objectif initial du travail ni à satisfaire l'attente qu'il génère chez le lecteur. Subsiste une interrogation quant à l'aptitude d'Agent_Zéro de simuler des processus sociaux réels, ainsi que l'auteur le prétend. Au-delà de cette critique, Agent_Zéro représente un effort précieux qui peut donner lieu à une amélioration en s'appuyant sur des recherches théoriques et empiriques. La simplicité du modèle est considérée comme un facteur positif pour l'auteur, ce qui est vrai dans la mesure où le modèle peut être modifié pour représenter diverses situations. Cependant, cette stratégie de modélisation qui renvoie au principe KISS (Keep it simple, stupid!) est plutôt une limitation, car elle ne garantit pas la simulation de la complexité de phénomènes sociaux réels. Pour conclure, il s'agit d'un ouvrage qui mérite d'être lu avec un regard critique. 